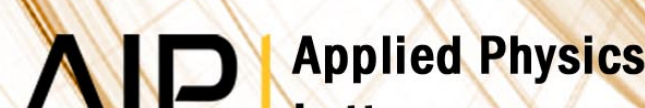 \\ Letters
}

\section{Scanning tunneling microscopy of the electronic structure of chemical vapor deposited diamond films}

\author{
J. M. Perez, C. Lin, W. Rivera, R. C. Hyer, M. Green et al.
}

Citation: Appl. Phys. Lett. 62, 1889 (1993); doi: 10.1063/1.109533

View online: http://dx.doi.org/10.1063/1.109533

View Table of Contents: http://apl.aip.org/resource/1/APPLAB/v62/i16

Published by the American Institute of Physics.

\section{Related Articles}

Contactless electroreflectance studies of Fermi level position on c-plane GaN surface grown by molecular beam epitaxy and metalorganic vapor phase epitaxy

Appl. Phys. Lett. 100, 181603 (2012)

Substrate effect on the electronic structures of CuPc/graphene interfaces

Appl. Phys. Lett. 100, 161603 (2012)

Magnetoexcitons and optical absorption of bilayer-structured topological insulators

Appl. Phys. Lett. 100, 161602 (2012)

High Eu $4 \mathrm{f} \mathrm{low-energy} \mathrm{oscillator} \mathrm{strength} \mathrm{in} \mathrm{the} \mathrm{isostructural} \mathrm{rare-earth} \mathrm{Zintl} \mathrm{compounds} \mathrm{Euln2X2} \mathrm{(X=P,As)}$

Appl. Phys. Lett. 100, 151906 (2012)

Modification of the surface-state occupancy on noble metal films with stacking fault arrays

Appl. Phys. Lett. 100, 141609 (2012)

\section{Additional information on Appl. Phys. Lett.}

Journal Homepage: http://apl.aip.org/

Journal Information: http://apl.aip.org/about/about_the_journal

Top downloads: http://apl.aip.org/features/most_downloaded

Information for Authors: http://apl.aip.org/authors

\section{ADVERTISEMENT}

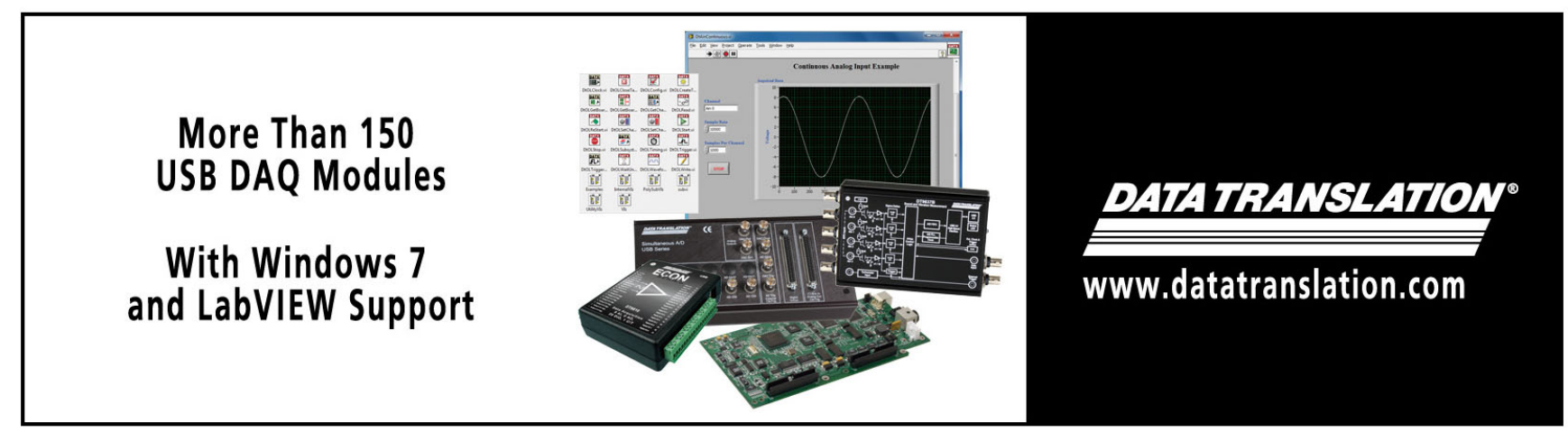




\title{
Scanning tunneling microscopy of the electronic structure of chemical vapor deposited diamond films
}

\author{
J. M. Perez, C. Lin, and W. Rivera \\ Department of Physics, University of North Texas, Denton, Texas 76203 \\ R. C. Hyer, M. Green, and S. C. Sharma \\ Department of Physics, The University of Texas at Arlington, Arlington, Texas 76019 \\ D. R. Chopra and A. R. Chourasia \\ Department of Physics, East Texas State University, Commerce, Texas 75429
}

(Received 18 August 1992; accepted for publication 22 February 1993)

\begin{abstract}
Scanning tunneling microscopy has been used to characterize the electronic structure and surface morphology of diamond films grown using the hot filament and microwave plasma chemical vapor deposition techniques. We observe a significant difference between the current-voltage $(I-V)$ curves for the two types of films. The $I-V$ curves for the hot-filament grown films are characterized by a well-defined zero-current region from which a surface band gap of $4.1 \mathrm{eV}$ is measured. The $I-V$ curves for the microwave plasma grown films exhibit a rectifying behavior which can be modeled by surface band bending. We compare the surface density of states obtained from the $I-V$ curves with those obtained from x-ray photoelectron and appearance potential spectroscopies.
\end{abstract}

Diamond films grown using the chemical vapor deposition (CVD) technique have recently attracted considerable interest due to the large number of potential applications of such films. ${ }^{1,2}$ Important applications include thinfilm transistors ${ }^{3}$ where detailed knowledge of the electronic properties of the surface of the film is crucial. Recently, scanning tunneling microscopy (STM) and atomic force microscopy (AFM) have been used to image CVD grown diamond films. ${ }^{4-6}$ In addition to imaging surfaces, STM can also be used to probe the local electronic properties of surfaces by measuring the tunneling current versus voltage, a technique referred to as tunneling spectroscopy ${ }^{7,8}$ In this letter, we present tunneling spectra and STM images of diamond films grown using the hot-filament and microwave plasma CVD techniques. For the hot-filament grown diamond films, we observe symmetric current-voltage ( $I$ $V$ ) curves with a well-defined surface band-gap region of approximately $4.1 \mathrm{eV}$. The surface density of states obtained from the tunneling spectra is found to be in good agreement with $\mathrm{x}$-ray photoelectron spectroscopy (XPS) and appearance potential spectroscopy (APS) data. For the microwave plasma grown films, we observe a rectifying $I-V$ curve which is modeled by band bending at the surface.

Diamond films were deposited by using hot-filament assisted CVD at low pressures ( $\sim 25$ Torr) using $0.25 \%$ $\mathrm{CH}_{4}$ in $\mathrm{CH}_{4} / \mathrm{H}_{2}$ mixtures. These films were deposited on $\mathrm{Si}(111)$ substrates maintained at about $1100 \mathrm{~K} .{ }^{9}$ The microwave plasma film was grown by using plasma enhanced CVD. The films were approximately $1 \mu \mathrm{m}$ thick. The Raman spectra of the hot filament and microwave plasma films show a sharp peak at $1332 \mathrm{~cm}^{-1}$ which is the characteristic Raman line for diamond. We have discussed detailed characteristics of the hot-filament grown films elsewhere. ${ }^{9}$ Both the hot filament and microwave plasma grown films contain predominantly $s p^{3}$ bonding; from the analysis of the Raman spectra, we estimate the $s p^{3} / s p^{2}$ bonding ratio to be 9.0 in both films. However, in the case of the microwave plasma grown film the $1332 \mathrm{~cm}^{-1}$ diamond line is significantly narrower. The full width at half maximum of the diamond lines are $4.15 \pm 0.02 \mathrm{~cm}^{-1}$ and $5.62 \pm 0.08 \mathrm{~cm}^{-1}$ for the microwave plasma and hotfilament grown films, respectively. Although the two films have about the same $s p^{3} / s p^{2}$ bonding ratio, the microwave plasma grown film contains much larger diamond domains.

Tunneling spectra and STM images were obtained using a commercial STM with a digital feedback loop made by Burleigh Instruments. ${ }^{10}$ The spectra and images were obtained in air using a mechanically cut Pt-Ir tip. Typical sample voltages and constant tunneling currents used for imaging were $3-4 \mathrm{~V}$ and $0.5-1.0 \mathrm{nA}$, respectively. The $I-V$ curves were obtained at a particular sample location by interrupting the feedback and measuring the tunneling current as a function of sample voltage for a fixed distance between the sample and tip. The tunneling current was measured using a 12 bit analog to digital converter. The surface density of states was computed from the $I-V$ curves using the expression for the normalized conductivity. ${ }^{8}$ To achieve a high signal to noise ratio in the $I-V$ curves several $I-V$ curves were measured at the same location and the results averaged. Tunneling current versus sample-tip separation $(I-S)$ measurements showed a strong dependence of the tunneling current on separation with a barrier height of approximately $5.0 \mathrm{eV}$, consistent with tunneling across a vacuum gap.

Figures 1(a) and 1(b) show a top view and corresponding three-dimensional topographic STM image of a $2 \times 2 \mu \mathrm{m}$ area of the hot-filament grown diamond film. Large crystallites on the order of $0.5 \mu \mathrm{m}$ are observed at this resolution. The film is relatively smooth with a rootmean-squared roughness of $0.29 \mu \mathrm{m}$. At higher resolution, microcrystallites measuring approximately $50 \mathrm{~nm}$ in width and $50 \mathrm{~nm}$ in height are observed, as shown in Figs. 1(c) 
(a)
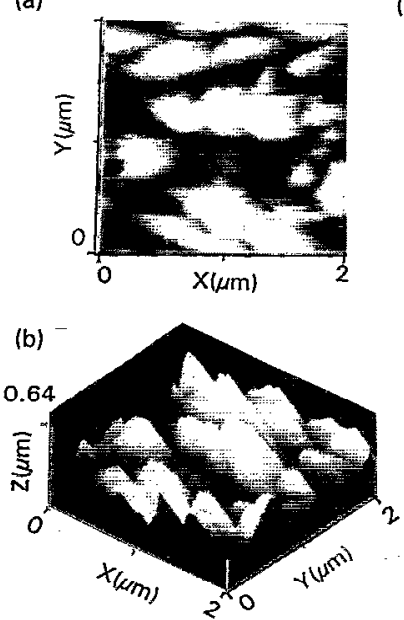

(c)

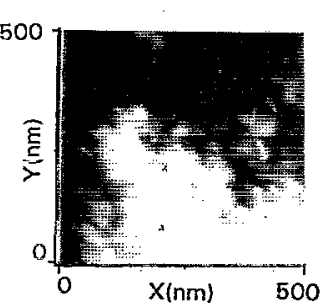

(d)

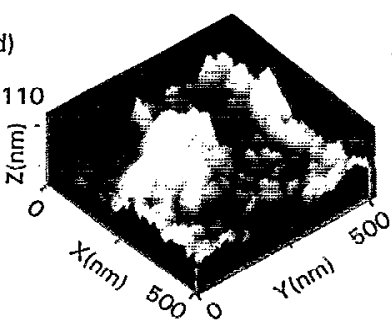

(e)
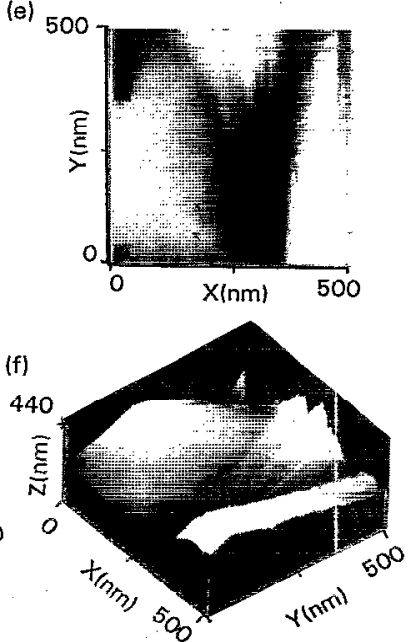

FIG. 1. (a) Top view STM image of the hot-filament grown diamond film, (b) three-dimensional topographic image of (a). (c) Higher resolution top view STM image of the hot-filament grown diamond film. X's denote locations where tunneling spectroscopy data was obtained, (d) three-dimensional topographic image of (c), (e) top view STM image of microwave plasma grown diamond film. X's denote locations where tunneling spectroscopy data was obtained, $(f)$ three-dimensional topographic image of $(e)$.

and 1(d). These findings are in agreement with high resolution transmission electron microscope images of the same film. ${ }^{11}$ Figures 1(e) and 1(f) show a top view STM image and corresponding three-dimensional topographic image of a $500 \times 500 \mathrm{~nm}$ area of the microwave plasma grown film. The crystallites are larger than in the hot-filament grown film. The smallest crystallites observed measured approximately $500 \mathrm{~nm}$ in width and $500 \mathrm{~nm}$ in height, as shown in Fig. 1(f).

$I-V$ curves were measured at the sample locations indicated in Figs. 1(c) and 1 (e) and other locations. The $I-V$ curves measured at different locations were similar. Figure 2(a) shows a typical $I-V$ curve for the hot-filament grown diamond film. The tunneling current is symmetric and has a well-defined zero-current region characteristic of a band gap. Similar $I-V$ curves having a zero-current region have been reported for $\mathrm{Si}$ and GaAs. ${ }^{12,13}$ The surface band gap of the diamond film measured from the width of the zero-current region in Fig. 2 (a) is $4.1 \mathrm{eV}$, to be compared with the bulk band gap of a diamond of $5.45 \mathrm{eV}$. Figure 2(b) shows a typical $I-V$ curve for the microwave plasma grown diamond film. The $I-V$ curves in this case show a tunneling current only for negative sample voltages; a characteristic of rectifying behavior. The same rectifying behavior was observed using platinum, tungsten, and gold tips, making tip-induced band bending unlikely. Similar rectifying $I-V$ curves have been reported for $\mathrm{ZnO}{ }^{14}$ hydrogen-terminated $\mathrm{Si}(111),{ }^{15}$ and sulfur-terminated GaAs. ${ }^{16}$ The rectification is attributed to surface band bending and the forward current is well modeled by the expression $^{14-16}$

$$
J=J_{0}\left\{\exp \left[q\left(V_{B 0}-V_{B i}\right) / k T-1\right]\right\}
$$

for the forward current of a Schottky barrier where $V_{B 0}$ is the barrier height and $V_{B i}=V_{B 0}\left\{\left[(c+1)^{2}-V / V_{B 0}\right]^{1 / 2}\right.$ $-c\}^{2}$ with $c$ a constant is the band bending as a function of sample voltage. Figure 2(c) shows a fit to the forward current shown in Fig. 2(b) using the above expression showing good agreement with the model. The band bending in $\mathrm{ZnO}$ is attributed to water on the surface, and in $\mathrm{Si}$ and GaAs to hydrogen and sulfur termination, respectively. Rectifying point contacts on diamond films have been reported to produce larger rectification in diamond films with larger diamond domains. ${ }^{17}$

To obtain the surface density of states, $\rho$, of the hotfilament grown diamond film from the $I-V$ curves we used the expression ${ }^{8}$

$$
\rho \propto \frac{d I / d V}{I / V}
$$

where the right-hand side is called the normalizcd conductivity. This expression is valid for nonzero tunneling current $I$ but is divergent in the band-gap region where $I$ is zero. The singularity is removed by setting $\rho$ equal to zero in the band-gap region, or using an average value for $I / V$ which is nonzero in the band-gap region. ${ }^{8,18} \mathrm{We}$ used both normalization techniques and obtained similar results. Figure 3 shows $\rho$ as a function of $V$ computed from the $I-V$ curve shown in Fig. 2(a) using the former normalization technique. The peaks at -2.8 and $3.7 \mathrm{eV}$ are attributed to overshoot from the numerical differentiation since these peaks are not observed in XPS or APS data as discussed below. This type of overshoot occurs when computing the normalized conductivity from $I-V$ curves. ${ }^{10}$ The peaks in the valence band observed at -6.1 and $-8.1 \mathrm{eV}$ are in good agreement with XPS data for the same film. ${ }^{19}$ A peak in the conduction band is observed at $7.0 \mathrm{eV}$. To determine the conduction band surface density of states of the sample we analyzed the sample using APS. ${ }^{20}$ The APS data showed a peak at $6.5 \mathrm{eV}$ above the Fermi level which correlates well with the $7.0 \mathrm{eV}$ peak in the STM surface density of states.

In summary, we have studied the electronic structure and surface morphology of CVD diamond films using STM. Hot-filament grown films containing microcrystal- 



FIG. 2. (a) Tunneling current vs sample voltage for the hot-filament grown diamond film. The zero-current region corresponds to a surface band gap of approximately $4.1 \mathrm{eV}$. (b) Tunneling current vs sample voltage for the microwave plasma grown diamond film. (c) Data show semilog plot of the $I-V$ curve of the microwave plasma grown film shown in (b). Solid line shows fit to the forward current part of the $I-V$ curve using the expression in the text for the forward current of a Schottky barrier with a barrier height of $4 \mathrm{~V}$.

lites showed a well-defined surface band gap and a surface density of states in agreement with XPS and APS. The microwave plasma grown films with larger crystallites and larger domains, as determined from Raman spectroscopy, exhibited rectifying behavior in good agreement with a simple model for surface band bending. We are currently applying the tunneling spectroscopy technique to study the nucleation and initial stages of growth of diamond films.

We thank Dr. Earl W. Stromberg for the plasma grown diamond film. This work was supported in part by

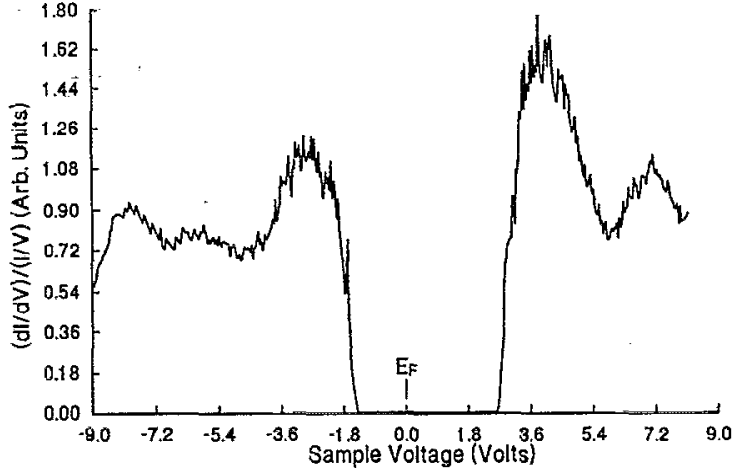

FIG. 3. Surface density of states of the hot-filament grown diamond film obtained from the $I-V$ curve shown in Fig. 2(a). Peaks corresponding to occupied states at -6.1 and $-8.1 \mathrm{eV}$ are in agreement with $\mathrm{x}$-ray photoelectron spectroscopy data. The peak at $7.0 \mathrm{eV}$ is in agreement with appearance potential spectroscopy.

the National Science Foundation under Award No. DMR9112940. Work at East Texas State University was supported in part by the Robert A. Wellch Foundation Grant No. T-643.

${ }^{1}$ B. V. Derjaguin, D. V. Fedoseev, N. D. Polyanskaya, and E. V. Statenkova, Krystallografiya 21, 433 (1976).

${ }^{2}$ W. A. Yarbrough and R. Messier, Science 242, 688 (1990).

${ }^{3}$ J. Mort, M. A. Machonkin, and K. Okumura, Appl. Phys. Lett. 59, 455 (1992).

${ }^{4}$ H.-G. Busman, H. Sprang, I. V. Herlel, W. Zimmermann-Edling, and H.-J. Guntherodt. Appl. Phys. Lett. 59, 295 (1992).

${ }^{5}$ V. Baranauskas, M. Fukui, C. R. Rodrigues, N. Parizotto, and V. J. Trava-Airoldi, Appl. Phys. Lett. 60, 1567 (1992).

${ }^{6}$ L. F. Sutcu, M. S. Thompson, C. J, Chu, R. H. Haugue, J. L. Margrave, and M. P. D'Evelyn, Appl. Phys. Lett. 60, 1685 (1992).

${ }^{7}$ F. Salvan, H. Fuchs, A. Baratoff, and G. Binning, Surf. Sci. 162, 634 (1985).

${ }^{8}$ R. M Feenstra, in Scanning Tunneling Microscopy and Related Methads, edited by R. J. Behm, N. Garcia, and R. Rohrer (Kluwer, Boston, 1990), p. 211.

${ }^{9}$ S. C. Sharma, M. Green, R. C. Hyer, C. A. Dark, T. D. Black, A. R. Chourasia, D. R. Chopra, and K. K. Mishra, J. Mater. Res. 5, 2424 (1990).

${ }^{10}$ Burleigh Instruments, Inc., Fishers, NY 14453.

${ }^{11}$ R. F. Pinizzotto (private communication).

${ }^{12}$ J. A. Stroscio, R. M. Feenstra, and A. P. Fein, Phys. Rev. Lett. 58, 1192 (1987).

${ }^{13}$ R. M. Feenstra and J. A. Stroscio, J. Vac. Sci. Technol. B 5, 923 (1987).

${ }^{14}$ G. S. Rohrer and D. A. Bonnell, J. Vac. Sci. Technol. B 9, 783 (1991).

${ }^{15}$ W. J. Kaiser, L. D. Bell, M. H. Hecht, and F. J. Grunthaner, J. Vac. Sci. Technol. A 6, 519 (1988).

${ }^{16}$ V. L. Berkovits, L. F. Ivantsov, I. V. Makarenko, T. V. L'vova, R. V. Khasieva, and V. I. Safarov, Sov. Phys. Semicond. 25, 231 (1991).

${ }^{17}$ K. Miyata, Y. Matsui, K. Kumagai, S. Miauchi, K. Kobashi, and A. Nakaue, in New Diamond Science and Technology, edited by R. Messier, J. T. Glass, J. E. Butler, and R. Roy (Materials Research Society, Pittsburgh, 1991), p. 981.

${ }^{18}$ P. Martensson and R. M. Feenstra, Phys. Rev. B 39, 7744 (1989).

${ }^{19}$ A. R. Chourasia, D. R. Chopra, S. C. Sharma, M. Green, C. A. Dark, and R. C. Hyer, Thin Solid Films 193/194, 1079 (1990).

${ }^{20}$ D. R. Chopra and A. R. Chourasia, Scanning Microscopy 2, 677 (1988). 